\title{
Einsatz von Hefederivaten zur Steigerung der phenolischen Reife und Aromaintensität von Wein
}

\author{
Impact of yeast derivatives to increase the phenolic maturity and aroma intensity of wine \\ L'impatto di lieviti inattivati all'aumento della maturazione fenolica e intensità aromatica del vino
}

\author{
Christoph Patauner ${ }^{1}$, Christof Sanoll ${ }^{1}$ \\ ${ }^{1}$ Versuchszentrum Laimburg, Pfatten (BZ), Italien
}

\section{ABSTRACT}

To increase the aroma of white wine through viticultural or oenological methods is a challenge for every wine producer. The same applies to the increase in phenolic maturity and color intensity in red wine. These two requirements play a decisive role in vintages for which there is a risk of growing grapes with insufficient ripeness. In the wine industry, products made from selected yeast derivatives have recently been offered. These specially developed yeast derivatives are applied two times to the entire foliage. The first, when the berries soften and the second at an interval of 10-14 days. What exactly is caused by these yeast derivatives is not fully recognized. According to the manufacturer, they should generally intensify the wine aroma of white grape varieties and improve the phenolic ripeness of red grape varieties. With this project, this possibility of increasing the aroma and improving the phenolic structure was tested and the extent of the change was measured. The product LaIVigne ${ }^{\circledast}$ Aroma from Lallemand was tested on neutral and aromatic white wine varieties, while the product LaIVigne ${ }^{\circledR}$ Mature from Lallemand was tested on structured red wine varieties.

\section{KEYWORDS}

LalVigne ${ }^{\circledR}$ Aroma, LalVigne ${ }^{\circledR}$

Mature, yeast derivatives, phenolic maturity, aroma intensity of wine

\section{CITE ARTICLE AS}

Patauner Christoph, Sanoll Christof, Surname here Forename here et.al. (2021). Impact of yeast derivatives to increase the phenolic maturity and aroma intensity of wine. Laimburg Journal 03/2021 DOI: $10.23796 / \mathrm{LJ} / 2021.007$

\section{CORRESPONDING AUTHOR}

Christoph Patauner

Laimburg 6, Pfatten, I-39040 Auer (BZ), Italien

christoph.patauner@laimburg.it +390471969626 


\section{EINFÜHRUNG}

Nach wie vor ist die Steigerung des Aromas durch weinbauliche oder önologische Maßnahmen beim Weißwein eine Herausforderung für die Weinproduzenten. Dasselbe gilt für die Steigerung der phenolischen Reife und Farbausbeute bei Rotwein. Ganz speziell spielen diese beiden Ansprüche in Jahrgängen, die Gefahr laufen, Trauben mit ungenügender Reife wachsen zu lassen, eine entscheidende Rolle. In der Weinwirtschaft sind jüngst Produkte aus selektionierten $\mathrm{He}$ federivaten im Angebot. Diese speziell entwickelten Hefederivate werden mit zwei Spritzungen auf die gesamte Laubwand, die erste bei Weichwerden der Beeren und die zweite im Abstand von 10-14 Tagen, direkt im Weinberg angewendet. Welche Wirkung genau durch diese Hefederivate hervorgerufen wird, ist noch nicht ganz geklärt. Laut Hersteller aber sollen sie grundsätzlich bei weißen Traubensorten das Weinaroma intensivieren und bei roten Traubensorten die phenolische Reife verbessern. Dieses Projekt untersucht die Möglichkeit der Aromasteigerung und die Verbesserung der Phenolstruktur und misst das Ausmaß dieser Veränderungen. Das Produkt LaIVigne ${ }^{\circledR}$ Aroma von Lallemand wurde dabei auf neutralen und aromatischen Weissweinsorten getestet, das Produkt LalVigne ${ }^{\circledR}$ Mature von Lallemand hingegen auf schweren Rotweinsorten.

\section{METHODEN}

Der Versuch wurde in 2 aufeinanderfolgenden Jahren durchgeführt. Dazu wurden Ertragsanlagen in klassischen Südtiroler Weinbauzonen ausgewählt. Im Jahr 2017 wurde dazu das Produkt LalVigne ${ }^{\circledR}$ Aroma auf Riesling getestet. Im Jahr 2018 wurde das Produkt LalVigne ${ }^{\circledast}$ Aroma auf Weissburgunder und Sauvignon Blanc sowie das Produkt LalVigne ${ }^{\circledast}$ Mature auf Merlot und Lagrein versucht. Die Produkte selbst wurden uns dabei vom Hersteller (Lallemand Italia) zur Verfügung gestellt. Anwendung, Ausbringung und Einsatzzeitpunkt erfolgten exakt laut den Angaben des Herstellers (Tab. 1).

Innerhalb einer Anlage wurde das jeweilige Produkt in randomisierten Blöcken ausgebracht. Alle Anlagen befanden sich in homogenem Zustand. Das Wachstum aller Rebstöcke war ruhig und ausgeglichen. Alle Blöcke hatten dasselbe Ertragsniveau. Die agronomische Bewirtschaftung jeder Anlage erfolgte "betriebsüblich". Bis auf die Spritzungen mit den zu testenden Hefederivaten wurden alle Blöcke komplett identisch und vergleichbar bewirtschaftet.

\section{ÖNOLOGIE}

Die Trauben wurden am entsprechenden Lesezeitpunkt von Hand geerntet und in kleinen Kisten (18 kg Fassungsvermögen) in den Kellereibetrieb des Versuchszentrums Laimburg gebracht (Tab. 2).

Von jeder Rebsorte wurden zum selben Moment je 2 Wiederholungen der "behandelten Variante" geerntet und jeweils 2 Wiederholungen der "unbehandelte Kontrolle". Die Weinbereitung erfolgte nach dem Protokoll, welches die Firma "Lallemand Italia" für diesen Versuch empfohlen hat.

\section{WEISSWEINBEREITUNG}

Jede einzelne Wiederholung umfasste $50 \mathrm{~kg}$ an Lesegut. Die Trauben wurden mit einer Abbeermaschine des Typs CMA Lugana $1 R$ (Stundenleistung 4-6 t/h) leicht angequetscht (ohne Stachelwalze) aber nicht abgebeert. Es wurde keine Maischestandzeit durchgeführt. Das angequetschte Traubenmaterial wurde händisch in die vollautomatisch betriebenen Pressen des Typs Europress Modell T1 mit 100 L Fassungsvermögen gegeben und daraufhin mit einem Automatikprogramm gepresst. Dabei kamen zwei verschiedene Druckstufen zum Einsatz. Begonnen wurde mit einem Druck von 1 bar für 10 Minuten, woraufhin zwei Wiederholungen von je 2 bar für 10 Minuten folgten. Der Rücksetzdruck belief sich auf 0,05 bar. Zwischen jeder Druckstufe wurde der aufgebaute Druck automatisch abgebaut, der Tresterkucher ausreichend gescheitert und mit der nächsten Druckstufe fortgefahren. Der ablaufende Saft wurde zur Gänze in der Mostauffangwanne gesammelt. $40 \%$ der zu erwartenden Zielmenge wurden unverzüglich mit 30 mg/l Kaliummetabisulfit (E 224) behandelt. Nach Beendigung des automatischen Presszyklus wurde der Saft in einer Korbflasche mit $34 \mathrm{~L}$ Fassungsvermögen gesammelt und die Gesamtmenge schliesslich mit 30 mg/l Kaliummetabisulfit (E 224) behandelt bzw. nachgeschwefelt. Der Most wurde für 16 Stunden bei $5{ }^{\circ} \mathrm{C}$ statisch entschleimt, im Anschluss der klare Überstand abgezogen und der Entschleimungstrub entfernt, bevor den Mosten $15 \mathrm{~g} / \mathrm{hl}$ Lysozym (Lallemand) zugesetzt wurden. Der Most wurde vorsichtig auf $20{ }^{\circ} \mathrm{C}$ erwärmt und je Wiederholung mehrere Mostproben entnommen, um diese auf die wichtigsten Mostinhaltsstoffe sowie Aromen zu untersuchen. $25 \mathrm{~g} / \mathrm{hl}$ Trockenreinzuchtefe der Art Saccharomyces Cerevisiae LalvinQA23 Yseo (Lallemand Italia) wurden nach Herstellerangaben rehydriert und der Most damit inokuliert. Die alkoholische Gärung erfolgte bei konstanten $21^{\circ} \mathrm{C}$ über regulierte Raumtemperatur. Dem Most wurden zweimal $20 \mathrm{~g} / \mathrm{hl}$ des Gärsalzes Fermaid E (Lallemand Italia) zugeführt. Der erste Abstich erfolgte unbelüftet bei Gärungsstillstand bzw. bei Restzuckergehalt $<4,0 \mathrm{~g} / \mathrm{l}$. Im Anschluss erfolgte eine kurzzeitige Lagerung in der Kühlzelle bei $5{ }^{\circ} \mathrm{C}$ für ca. 10 Tage mit anschließender Schwefelung von $30 \mathrm{mg} / \mathrm{l}$ Kaliummetabisulfit (E 224). Nach einem weiteren Abzug wurden die Weine bei einer Temperatur zwischen 14 und $16^{\circ} \mathrm{C}$ gelagert. Während der Weinlagerung wurde ein Gehalt an freier schwefeliger Säure von $25 \mathrm{mg} / \mathrm{l}$ eingestellt und überwacht. Die Füllung erfolgte in 0,5 L Glasflaschen mit 30/60 BVS Mündung nach unmittelbar vorhergehender Vor-, Blankund Sterilfiltration $(0,45 \mu \mathrm{m})$. Als Verschluss wurde Stelvin 30/60 BVS, Dichtungsscheibe Stanniol, gewählt.

\section{ROTWEINBEREITUNG}

Jede einzelne Wiederholung umfasste $50 \mathrm{~kg}$ an Lesegut. Die Trauben wurden mit der Abbeermaschine des Typs CMA Lugana $1 R$ (Stundenleistung 4-6 t/h) abgebeert. Die Maische wurde daraufhin in Weithalskorbflaschen mit $34 \mathrm{~L}$ Fassungsvermögen gegeben und mit $20 \mathrm{mg} / \mathrm{l}$ Kaliummetabisulfit (E 224) geschwefelt. Es wurden je Wiederholung mehrere Mostproben entnommen, um diese auf die wichtigsten Mostinhaltsstoffe sowie Aromen zu untersuchen. In Folge wurden die Maischen mit $20 \mathrm{~g} / \mathrm{hl}$ Trockenreinzuchthefe des Typs Saccharomyces cerevisiae LalvinICV D254 Yseo (Lallemand Italia) beimpft. Die Temperaturführung während der alkoholischen Gärung erfolgte über ein automatisches temperaturgesteuertes System (WFT VinPilot Pro) und war in jeder Korbflasche identisch. Der Maische wurden zweimal $20 \mathrm{~g} / \mathrm{hl}$ des Gärsalzes Fermaid E (Lallemand Italia) und 24 Stunden nach dem Hefezusatz Milchsäurebakterien Oenococus oeni VP41 MBR (Lallemand Italia) zugesetzt. Der erste Abstich erfolgte belüftend über ein Kupfersieb ohne Schwefelung (E 224). Die vergorene Maische wurde schonend bei 0,5 bar für 10 Minuten in den vollautomatisch betriebenen Pressen des Typs Europress Modell T1 mit 100 L Fassungsvermögen, Rücksetzdruck 0,05 bar, ausgepresst. 
Der Presswein wurde zu 100\% dem Wein beigemengt. Der zweite Abzug erfolgte 24 Stunden später mit Zugabe von 30 mg/l Kaliummetabisulfit (E224). Die Weine lagerten bei einer Temperatur zwischen 14 und $16^{\circ} \mathrm{C}$. Während der Weinlagerung wurde ein Gehalt an freier schwefeliger Säure von 20$25 \mathrm{mg} / \mathrm{l}$ eingestellt und überwacht. Die Füllung erfolgte in 0,5-Liter-Glasflaschen mit 30/60 BVS Mündung nach unmittelbar vorhergehender Vor-, Blank- und Sterilfiltration $(0,45 \mu \mathrm{m})$. Als Verschluss wurde Stelvin 30/60 BVS, Dichtungsscheibe Stanniol, gewählt.

\section{ANALYSEN}

Das Labor für Wein- und Getränkeanalytik des Versuchszentrums Laimburg hat mittels FTIR-Analysen (FOSS ${ }^{\circledR}$, WineScan ${ }^{\mathrm{TM}}$ ) sowie mittels eines multiparametrischen Analyseautomaten (Hyperlab Plus, Steroglass) gemäß interner Methodenvorschriften folgende Parameter auf Most und Wein bestimmt (Abb. 1):

- pH-Wert (Most und Wein)

- Gesamtsäure g/l ausgedrückt als Weinsäure (Most und Wein)

- Zuckergradation ${ }^{\circ} \mathrm{KMW}$ (Most)

- Flüchtige Säure g/L ausgedrückt als Essigsäure (Wein)

- Restzucker g/l (Wein)

- Gesamtextrakt g/l (Wein)

- Alkohol \% vol (Wein)

- Weinsäure g/l (Wein)

- Apfelsäure g/l (Wein)

- Hefeverwertbarer Stickstoff mg/l (Most)

- Ammoniumstickstoff mg/l (Most)

- Alfa-Aminosäurestickstoff mg/l (Most)

Außerdem wurden von allen Mosten und Weinen des Jahrgangs 2018 Aromaanalysen im Laboratorio Analisi Agroalimentari der Fondazione Edmund Mach in San Michele all'Adige durchgeführt (Tab. 3).

\section{WEINSENSORIK}

Alle Weine wurden zweimal verkostet. Einmal als Jungwein ca. 6 Monate nach der Einkellerung, das zweite Mal als gereifter Wein ca. 18 Monate ab Einkellerung. Gelagert wurden die abgefüllten Flaschen im Weinarchiv der Laimburg bei konstanten $14{ }^{\circ} \mathrm{C}$. In Tabelle 4, Tabelle 5 und Tabelle 6 sind die bewerteten Attribute dargestellt.

Für die sensorische Charakterisierung wurde ein Verkostungsprofil nach Weiß 1972 (modifiziert - nicht publiziert) verwendet. Die Daten wurden dabei mit der Software FIZZ

\section{ZUSAMMENFASSUNG}

Nach wie vor ist die Steigerung des Aromas durch weinbauliche oder önologische Maßnahmen beim Weißwein eine Herausforderung für die Weinproduzenten. Dasselbe gilt für die Steigerung der phenolischen Reife und Farbausbeute bei Rotwein. Ganz speziell spielen diese beiden Ansprüche in Jahrgängen, die Gefahr laufen, Trauben mit ungenügender Reife wachsen zu lassen, eine entscheidende Rolle. In der Weinwirtschaft sind jüngst Produkte aus selektionierten Hefederivaten im Angebot. Diese speziell entwickelten Hefederivate werden mit zwei Spritzungen auf die gesamte Laubwand, die erste bei Weichwerden der Beeren und die zweite im Abstand von 10-14 Tagen, direkt im Weinberg angewendet. Welche Wirkung genau durch diese Hefederivate hervorgerufen wird ist noch nicht ganz geklärt. Laut Hersteller aber sollen sie grundsätzlich bei weißen Traubensorten das Weinaroma intensivieren und bei roten Traubensorten die phenolische Reife verbessern. Durch dieses Projekt wurde diese Möglichkeit der Aromasteigerung und Verbesserung der PhenolStruktur angewandt und das Ausmaß der Veränderung gemessen. Das Produkt LalVigne ${ }^{\circledR}$ Aroma von Lallemand wurde dabei auf neutralen und aromatischen Weissweinsorte getestet, das Produkt LalVigne ${ }^{\circledR}$ Mature von Lallemand hingegen auf schweren Rotweinsorten.

\section{RIASSUNTO}

Aumentare l'aroma di vini bianchi attraverso provvedimenti viticoli o enologici rappresenta tuttora una sfida per i produttori di vino. Lo stesso vale per l'aumento della maturità fenolica e l'aumento del colore nel vino rosso. Particolarmente nelle annate scadenti, dove l'uva fatica ad arrivare alla maturazione ottimale i parametri sopraindicati svolgono un ruolo cruciale.Di recente si trovano sul mercato dei prodotti di derivati da lievito selezionato.Questi derivati, appositamente sviluppati da lieviti, vengono applicati direttamente sulle vigne con due trattamenti sull'intera parete fogliare. II primo trattamento viene eseguito all'invaiatura, il secondo dopo un intervallo di 10-14 giorni. L'effetto preciso che crea l'uso di questi derivati non è stato del tutto chiarito. Secondo la ditta produttrice dovrebbe fondamentalmente intensificare l'aroma del vino delle varietà di uva a bacca bianca e migliorare la maturità fenolica delle varietà di uve a bacche rosse.II prodotto LaIVigne ${ }^{\circledR}$ Aroma di Lallemand viene testato su delle varietà d'uva a bacca bianca neutra ed aromatica, mentre il prodotto LalVigne ${ }^{\circledR}$ Mature di Lallemand su delle varietà di uva a bacca rossa destinata a produrre vini rossi strutturati.

by Biosystems erhoben. Um die Urteilssicherheit der einzelnen Panelmitglieder zu überprüfen, wurde das Verfahren nach Kobler 1996 angewandt. Dabei wurde für jeden einzelnen Parameter die Kontroll-Algorithmen durchgerechnet. Das Verkosterpanel setzte sich aus Technikern, Beratern und Fachleuten aus Weinbau und Kellerwirtschaft zusammen. Für die Auswertung der erhobenen Daten wurden die Programme
Microsoft ${ }^{\circledast}$ Office Excel 2013 von (CMicrosoft Corporation 1993-2001, für die statistische Auswertung IBM ${ }^{\circledR}$ SPSS $^{\circledR}$ Statistics Version 20 Release 20.0.0 für varianzanalytische Mittelwertvergleiche (Oneway ANOVA) oder multivarianter Varianzanalyse (Manova) verwendet. Als Post-Hoc-Test wurde Tukey-B mit Signifikanzniveau 0,05 über SPSS gerechnet (Abb. 2). 


\section{ERGEBNISSE}

\section{ERGEBNISSE ZUM PRODUKT LAL- VIGNE ${ }^{\circledR}$ AROMA}

In Bezug auf Wein- und Mostanalysen gibt es sortenübergreifend zwischen den behandelten Varianten LaIVigne ${ }^{\circledR}$ Aroma und den unbehandelten Kontrollen keine Unterschiede. Lediglich beim Restzuckergehalt ist ein vernachlässigbarer Unterschied in den Weinen festzustellen, wobei die unbehandelten Kontrollen etwas trockener ausgegoren sind als die behandelten Varianten LaIVigne ${ }^{\circledR}$ Aroma (Abb. 1).

Die Weinverkostungen haben außerdem ergeben, dass es zu keiner Steigerung oder Verbesserung des Aromas kommt, anders als es der Hersteller verspricht. Die Weine sind faktisch gleich. Bezugnehmend auf die Verkostungsparameter Fülle, Harmonie und Gesamteindruck schneidet die unbehandelte Kontrolle sogar signifikant besser ab als die Varianten behandelt LalVigne ${ }^{\circledast}$ Aroma (Abb. 3, Abb. 4, Abb. 5, Abb. 6).

Dass es zu keiner Steigerung oder Verbesserung bzw. Veränderung des Aromas durch den Einsatz des Produktes LalVigne ${ }^{\circledast}$ Aroma kommt, verdeutlichen auch die umfangreichen Aromaanalyse. Es konnten keinerlei Unterschiede gemessen werden, weder im Traubenmost noch im Wein.

\section{ERGEBNISSE ZUM PRODUKT LAL- VIGNE ${ }^{\circledR}$ MATURE}

In Bezug auf Wein- und Mostanalysen gibt es sortenübergreifend zwischen behandelt LalVigne $^{\circledR}$ Mature und den unbehandelten Kontrollen keine Unterschiede. Die Weinverkostungen haben ergeben, dass es zu keiner Steigerung oder Verbesserung der phenolischen Reife kommt. Die Weine sind faktisch gleich. Dies veranschaulicht der Vergleich der Verkostungsparameter Gerbstoffquantität, Bitterkeit der Gerbstoffe, Adstringenz und Weichheit am Gaumen. (Abb. 7, Abb. 8, Abb. 9, Abb. 10).

Bei den umfangreichen Aroma-Analysen auf Most und Wein sind bei 2 Aromen signifikante Unterschiede aufgetreten. Beim Aroma "Nonanoic acid“ (gemessen im Traubenmost) ist die unbehandelte Kontrolle signifikant höher. Beim Aroma „Ethyl pentanoate" (gemessen im Wein) ist die behandelte Variante LaIVigne ${ }^{\circledR}$ Mature signifikant höher. In Summe sind diese beiden Unterschiede aber zu vernachlässigen, da die Weinsensorik keine Unterschiede hervorbringen konnte (Abb. 11, Abb. 12).

\section{DISKUSSION}

Zusammenfassend kann gesagt werden, dass die getesteten Produkte LaIVigne ${ }^{\circledR}$ Aroma und LalVigne ${ }^{\circledR}$ Mature sortenübergreifend keinerlei Wirkung zeigen und weder bei weißen Traubensorten das Weinaroma intensivieren noch bei roten Traubensorten die phenolische Reife verbessern.

\section{DANKSAGUNG}

Ein Dank geht an Gerd Innerebner und Christian Roschatt von Arbeitsgruppe Mittelprüfung im Weinbau (Versuchszentrum Laimburg) für die Umsetzung des Freilandversuches. Ein weiterer Dank geht an Michael IIImer und Walter Gostner von der Agentur Landesdomäne für die Durchführung der Behandlungen im Freiland und die Pflege der Versuchsanlagen.

\section{REFERENCES}

[1] Weiss J., Willisch E., Knorr D. et al. (1972) Ergebnisse von Untersuchungen bezüglich der differenzierten Wirkung einer sensorischen bewertenden Prüfmethode gegenüber einer sensorischen Rangordnungs-Prüfmethode am Beispiel von Apfelsaft und Birnennektar. Confructa 17 (4/5), 237-250.
[2] Kobler A. (1996). La valutazione sensoriale dei vini ed il controllo degli assaggiatori mediante l'uso di schede di degustazione non strutturate. Rivista di Viticoltura e di Enologia 49 (4), 3-18.

[3] Flanzy M., Aubert S., Marinos M. (1969). New technique for determination of leucoanthocyanic tannins. Annales de technologie agricole 18, 327-328.
[4] Boulton R. (2001). The copigmentation of anthocyanins and its role in the color of red wine. A critical review. American Journal of Enology and Viticulture 52 (2), 67-87. 


\section{ANHANG 1: TABELLEN}

Tab. 1: Überblick der eingesetzten Produkte // Overview of the used products.

\begin{tabular}{|c|c|c|c|c|}
\hline Jahr & Sorte & Name Anlage & Ort Anlage & Produkt \\
\hline Year & Grape variety & Name of the vineyard & Location of the vineyard & Product Name \\
\hline 2018 & Weissburgunder & Sallmannhof & Meran & LalVigne ${ }^{\circledR}$ Aroma \\
\hline 2018 & Sauvignon Blanc & Fragsburg & Meran & LalVigne ${ }^{\circledR}$ Aroma \\
\hline 2018 & Lagrein & Prutznai & Kaltern & LalVigne ${ }^{\circledR}$ Mature \\
\hline 2018 & Merlot & Prutznai & Kaltern & LalVigne ${ }^{\circledR}$ Mature \\
\hline
\end{tabular}

Tab. 2: Lesezeitpunkt //Date of Harvest.

\begin{tabular}{ll}
$\begin{array}{l}\text { Sorte } \\
\text { Grape variety }\end{array}$ & $\begin{array}{l}\text { Lesezeitpunkt } \\
\text { Date of harvest }\end{array}$ \\
\hline Riesling & 28.09 .17 \\
\hline Weissburgunder & 11.09 .18 \\
\hline Sauvignon Blanc & 12.09 .18 \\
\hline Lagrein & 02.10 .18 \\
\hline Merlot & 10.10 .18 \\
\hline
\end{tabular}


Tab. 3: Überblick der durchgeführten Aromaanalysen // Overview to the aroma analysis.

\begin{tabular}{|c|c|c|}
\hline $\begin{array}{l}\text { Matrix } \\
\text { Matrix }\end{array}$ & $\begin{array}{l}\text { Art der Verbindung } \\
\text { Type of Compound }\end{array}$ & $\begin{array}{l}\text { Verbindung } \\
\text { Compound }\end{array}$ \\
\hline Traubenmost & Free Compounds (mg/L) & isobutyl acetate \\
\hline Traubenmost & Free Compounds (mg/L) & n-butyl acetate \\
\hline Traubenmost & Free Compounds (mg/L) & isopentyl acetate \\
\hline Traubenmost & Free Compounds (mg/L) & n-hexyl acetate \\
\hline Traubenmost & Free Compounds (mg/L) & 2-phenylethyl acetate \\
\hline Traubenmost & Free Compounds (mg/L) & ethyl phenyl acetate \\
\hline Traubenmost & Free Compounds (mg/L) & ethyl lactate \\
\hline Traubenmost & Free Compounds (mg/L) & ethyl isobutyrate \\
\hline Traubenmost & Free Compounds (mg/L) & ethyl isovalerate \\
\hline Traubenmost & Free Compounds (mg/L) & ethyl hexanoate \\
\hline Traubenmost & Free Compounds (mg/L) & ethyl octanoate \\
\hline Traubenmost & Free Compounds (mg/L) & ethyl decanoate \\
\hline Traubenmost & Free Compounds (mg/L) & diethyl-succinate \\
\hline Traubenmost & Free Compounds (mg/L) & isobutytric acid \\
\hline Traubenmost & Free Compounds (mg/L) & butanoic acid \\
\hline Traubenmost & Free Compounds (mg/L) & isovaleric acid \\
\hline Traubenmost & Free Compounds (mg/L) & valeric acid \\
\hline Traubenmost & Free Compounds (mg/L) & hexanoic acid \\
\hline Traubenmost & Free Compounds (mg/L) & octanoic acid \\
\hline Traubenmost & Free Compounds (mg/L) & nonanoic acid \\
\hline Traubenmost & Free Compounds (mg/L) & decanoic acid \\
\hline Traubenmost & Free Compounds (mg/L) & 1-hexanol \\
\hline Traubenmost & Free Compounds (mg/L) & trans-3-hexen-1-ol \\
\hline Traubenmost & Free Compounds (mg/L) & cis-3-hexen-1-ol \\
\hline Traubenmost & Free Compounds (mg/L) & 3-methylthio-1-propanol \\
\hline Traubenmost & Free Compounds (mg/L) & benzothiazole \\
\hline Traubenmost & Free Compounds (mg/L) & benzyl alcohol \\
\hline Traubenmost & Free Compounds (mg/L) & 2-phenylethanol \\
\hline Traubenmost & Free Compounds (mg/L) & linalol oxide $A$ \\
\hline Traubenmost & Free Compounds (mg/L) & linalol oxide B \\
\hline Traubenmost & Free Compounds (mg/L) & linalool \\
\hline Traubenmost & Free Compounds (mg/L) & alpha-terpineol \\
\hline Traubenmost & Free Compounds (mg/L) & terpinen-4-ol \\
\hline Traubenmost & Free Compounds (mg/L) & beta citronellol \\
\hline Traubenmost & Free Compounds (mg/L) & nerol \\
\hline Traubenmost & Free Compounds (mg/L) & geraniol \\
\hline Traubenmost & Free Compounds (mg/L) & geranic acid \\
\hline Traubenmost & Free Compounds (mg/L) & rose oxide I \\
\hline Traubenmost & Free Compounds (mg/L) & rose oxide II \\
\hline Traubenmost & Free Compounds (mg/L) & terpinolene \\
\hline Traubenmost & Free Compounds (mg/L) & guaiacol \\
\hline Traubenmost & Free Compounds (mg/L) & benzaldehyde \\
\hline Traubenmost & Free Compounds (mg/L) & methyl salicilate \\
\hline Traubenmost & Free Compounds (mg/L) & zingerone \\
\hline
\end{tabular}




\begin{tabular}{|c|c|c|}
\hline $\begin{array}{l}\text { Matrix } \\
\text { Matrix }\end{array}$ & $\begin{array}{l}\text { Art der Verbindung } \\
\text { Type of Compound }\end{array}$ & $\begin{array}{l}\text { Verbindung } \\
\text { Compound }\end{array}$ \\
\hline Traubenmost & Free Compounds (mg/L) & beta-damascone \\
\hline Traubenmost & Bound Compounds (mg/L) & 1-hexanol \\
\hline Traubenmost & Bound Compounds (mg/L) & trans-3-hexen-1ol \\
\hline Traubenmost & Bound Compounds (mg/L) & cis-3-hexen-1ol \\
\hline Traubenmost & Bound Compounds (mg/L) & 2-phenylethanol \\
\hline Traubenmost & Bound Compounds (mg/L) & benzyl alcohol \\
\hline Traubenmost & Bound Compounds (mg/L) & linalol oxide $A$ \\
\hline Traubenmost & Bound Compounds (mg/L) & linalol oxide B \\
\hline Traubenmost & Bound Compounds (mg/L) & linalool \\
\hline Traubenmost & Bound Compounds (mg/L) & alpha-terpineol \\
\hline Traubenmost & Bound Compounds (mg/L) & terpinen-4-ol \\
\hline Traubenmost & Bound Compounds (mg/L) & beta-citronellol \\
\hline Traubenmost & Bound Compounds (mg/L) & nerol \\
\hline Traubenmost & Bound Compounds (mg/L) & geraniol \\
\hline Traubenmost & Bound Compounds (mg/L) & geranic acid \\
\hline Traubenmost & Bound Compounds (mg/L) & rose oxide I \\
\hline Traubenmost & Bound Compounds (mg/L) & rose oxide II \\
\hline Traubenmost & Bound Compounds (mg/L) & benzaldehyde \\
\hline Traubenmost & Bound Compounds (mg/L) & methyl salycilate \\
\hline Traubenmost & Bound Compounds (mg/L) & zingerone \\
\hline Traubenmost & PRECURSORI TIOLICI & GSH - 3MH \\
\hline Traubenmost & PRECURSORI TIOLICI & Cys - $3 \mathrm{MH}$ \\
\hline Traubenmost & PRECURSORI TIOLICI & Cys - 4MMP \\
\hline Traubenmost & PRECURSORI TIOLICI & G-4mmp \\
\hline Wein & Free Compounds (mg/L) & isobutyl acetate \\
\hline Wein & Free Compounds (mg/L) & Ethyl butyrate + ethyl isobutyrate \\
\hline Wein & Free Compounds (mg/L) & ethyl-2-methylbuthyrate \\
\hline Wein & Free Compounds (mg/L) & n-butyl acetate \\
\hline Wein & Free Compounds (mg/L) & isopentyl acetate \\
\hline Wein & Free Compounds (mg/L) & ethyl isovalerate \\
\hline Wein & Free Compounds (mg/L) & ethyl pentanoate \\
\hline Wein & Free Compounds (mg/L) & ethyl hexanoate \\
\hline Wein & Free Compounds (mg/L) & n-hexyl acetate \\
\hline Wein & Free Compounds (mg/L) & ethyl heptanoate \\
\hline Wein & Free Compounds (mg/L) & ethyl lactate \\
\hline Wein & Free Compounds (mg/L) & 1-hexanol \\
\hline Wein & Free Compounds (mg/L) & rose oxide I \\
\hline Wein & Free Compounds (mg/L) & trans-3-hexen-1-ol \\
\hline Wein & Free Compounds (mg/L) & rose oxide II \\
\hline Wein & Free Compounds (mg/L) & cis-3-hexen-1-ol \\
\hline Wein & Free Compounds (mg/L) & ethyl octanoate \\
\hline Wein & Free Compounds (mg/L) & linalol oxide $A$ \\
\hline Wein & Free Compounds (mg/L) & linalol oxide B \\
\hline Wein & Free Compounds (mg/L) & benzaldehyde \\
\hline
\end{tabular}




\begin{tabular}{|c|c|c|}
\hline $\begin{array}{l}\text { Matrix } \\
\text { Matrix }\end{array}$ & $\begin{array}{l}\text { Art der Verbindung } \\
\text { Type of Compound }\end{array}$ & $\begin{array}{l}\text { Verbindung } \\
\text { Compound }\end{array}$ \\
\hline Wein & Free Compounds (mg/L) & isobutytric acid \\
\hline Wein & Free Compounds (mg/L) & linalool \\
\hline Wein & Free Compounds (mg/L) & terpinen-4-ol \\
\hline Wein & Free Compounds (mg/L) & butanoic acid \\
\hline Wein & Free Compounds (mg/L) & ethyl decanoate \\
\hline Wein & Free Compounds (mg/L) & isovaleric acid \\
\hline Wein & Free Compounds (mg/L) & diethyl-succinate \\
\hline Wein & Free Compounds (mg/L) & alpha-terpineol \\
\hline Wein & Free Compounds (mg/L) & 3-methylthio-1-propanol \\
\hline Wein & Free Compounds (mg/L) & valeric acid \\
\hline Wein & Free Compounds (mg/L) & beta citronellol \\
\hline Wein & Free Compounds (mg/L) & ethyl phenyl acetate \\
\hline Wein & Free Compounds (mg/L) & nerol \\
\hline Wein & Free Compounds (mg/L) & 2-phenylethyl acetate \\
\hline Wein & Free Compounds (mg/L) & methyl salicilate \\
\hline Wein & Free Compounds (mg/L) & beta-damascone \\
\hline Wein & Free Compounds (mg/L) & hexanoic acid \\
\hline Wein & Free Compounds (mg/L) & ethyl dodecanoate \\
\hline Wein & Free Compounds (mg/L) & geraniol \\
\hline Wein & Free Compounds (mg/L) & guaiacol \\
\hline Wein & Free Compounds (mg/L) & benzyl alcohol \\
\hline Wein & Free Compounds (mg/L) & 2-phenylethanol \\
\hline Wein & Free Compounds (mg/L) & benzothiazole \\
\hline Wein & Free Compounds (mg/L) & octanoic acid \\
\hline Wein & Free Compounds (mg/L) & nonanoic acid \\
\hline Wein & Free Compounds (mg/L) & decanoic acid \\
\hline Wein & Free Compounds (mg/L) & geranic acid \\
\hline Wein & Free Compounds (mg/L) & zingerone \\
\hline Wein & Free Compounds (mg/L) & terpinolene \\
\hline Wein & Bound Compounds (mg/L) & 1-hexanol \\
\hline Wein & Bound Compounds (mg/L) & trans-3-hexen-1ol \\
\hline Wein & Bound Compounds (mg/L) & cis-3-hexen-1ol \\
\hline Wein & Bound Compounds (mg/L) & 2-phenylethanol \\
\hline Wein & Bound Compounds (mg/L) & benzyl alcohol \\
\hline Wein & Bound Compounds (mg/L) & linalol oxide $A$ \\
\hline Wein & Bound Compounds (mg/L) & linalol oxide B \\
\hline Wein & Bound Compounds (mg/L) & linalool \\
\hline Wein & Bound Compounds (mg/L) & alpha-terpineol \\
\hline Wein & Bound Compounds (mg/L) & terpinen-4-ol \\
\hline Wein & Bound Compounds (mg/L) & beta-citronellol \\
\hline Wein & Bound Compounds (mg/L) & nerol \\
\hline Wein & Bound Compounds (mg/L) & geraniol \\
\hline Wein & Bound Compounds (mg/L) & geranic acid \\
\hline Wein & Bound Compounds (mg/L) & rose oxide I \\
\hline
\end{tabular}




\begin{tabular}{ccc}
$\begin{array}{c}\text { Matrix } \\
\text { Matrix }\end{array}$ & $\begin{array}{c}\text { Art der Verbindung } \\
\text { Type of Compound }\end{array}$ & $\begin{array}{c}\text { Verbindung } \\
\text { Compound }\end{array}$ \\
\hline Wein & Bound Compounds $(\mathrm{mg} / \mathrm{L})$ & rose oxide II \\
Wein & Bound Compounds $(\mathrm{mg} / \mathrm{L})$ & benzaldehyde \\
Wein & Bound Compounds $(\mathrm{mg} / \mathrm{L})$ & methyl salycilate \\
Wein & Bound Compounds $(\mathrm{mg} / \mathrm{L})$ & zingerone
\end{tabular}

Tab. 4: Verkostete Parameter bei Riesling // Tasted parameters on Riesling.

\begin{tabular}{ll}
$\begin{array}{l}\text { Kriterium } \\
\text { Criteria }\end{array}$ & $\begin{array}{l}\text { Beschreibung } \\
\text { Description }\end{array}$ \\
\hline Intensität im Geruch & wenig intensiv-sehr intensiv \\
\hline Reintönigkeit im Geruch & unsauber-reintönig \\
\hline Komplexität & einfach-komplex \\
\hline Typizität & untypisch-sehr typisch \\
\hline Reintönigkeit im Geruch Retronasal & unsauber-reintönig \\
\hline Fülle & dünn-voll \\
\hline Harmonie & unharmonisch-harmonisch \\
\hline Gesamteindruck & schlecht-ausgezeichnet \\
\hline
\end{tabular}


Tab. 5: Verkostete Parameter bei Weissburgunder und Sauvignon Blanc // Tasted parameters on Pinot Blanc and Sauvignon Blanc.

\begin{tabular}{|c|c|}
\hline Kriterium & Beschreibung \\
\hline Criteria & Description \\
\hline Intensität im Geruch & wenig intensiv-sehr intensiv \\
\hline Reintönigkeit im Geruch & unsauber-reintönig \\
\hline Komplexität & einfach-komplex \\
\hline Fruchtigkeit & wenig-viel \\
\hline Exotische Frucht & wenig-viel \\
\hline Zitrusfrucht & wenig-viel \\
\hline Blumige Noten & wenig-viel \\
\hline Grasige Noten & wenig-viel \\
\hline Würzige Noten & wenig-viel \\
\hline Reintönigkeit am Gaumen & unsauber-reintönig \\
\hline Typizität & untypisch-sehr typisch \\
\hline Fülle & dünn-voll \\
\hline Harmonie & unharmonisch-harmonisch \\
\hline Gesamteindruck & schlecht-ausgezeichnet \\
\hline
\end{tabular}

Tab. 6: Verkostete Parameter bei Merlot und Lagrein // Tasted parameters on Merlot and Lagrein.

\begin{tabular}{ll}
$\begin{array}{l}\text { Kriterium } \\
\text { Criteria }\end{array}$ & $\begin{array}{l}\text { Beschreibung } \\
\text { Description }\end{array}$ \\
\hline Farbe & zu hell-optimal-zu dunkel \\
\hline Intensität im Geruch & wenig intensiv-sehr intensiv \\
\hline Reintönigkeit im Geruch & unsauber-reintönig \\
\hline Komplexität & einfach-komplex \\
\hline Gerbstoffquantität & zu wenig-optimal-zu viel \\
\hline Gerbstoffe Hart & hart-rund \\
\hline Bitterkeit der Gerbstoffe & bitter-nicht bitter \\
\hline Adstringenz & adstringierend-nicht adstringierend \\
\hline Weichheit am Gaumen & hart-weich \\
\hline Typizität & unharmonisch-harmonisch \\
\hline Harmonie & dünn-voll \\
\hline Gesamteindruck & untypisch \\
\hline
\end{tabular}




\section{ANHANG 2: ABBILDUNGEN}

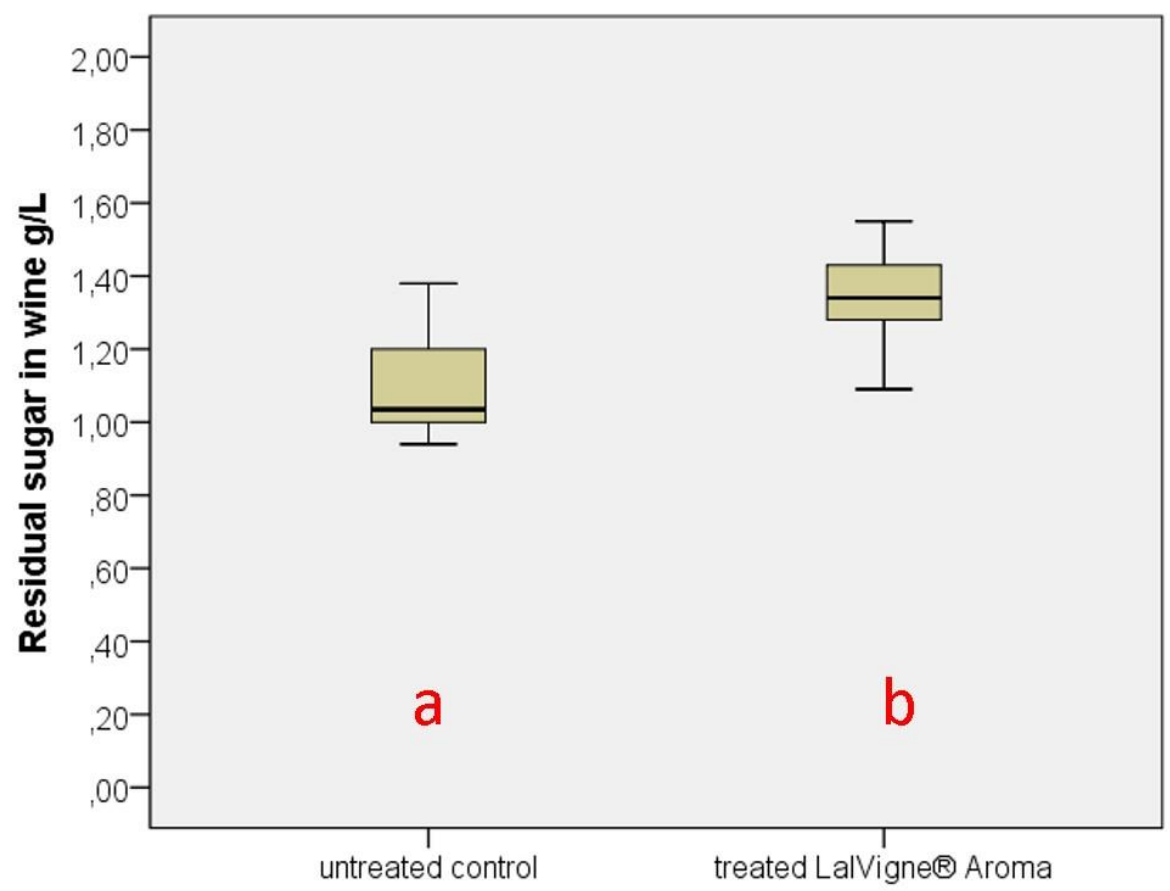

Abb. 1: Boxplot zum analytischen Parameter Restzuckergehalt im Wein (*Boxplots welche mit verschiedenen Buchstaben gekennzeichnet sind, unterscheiden sich mit dem Tukey-B Test bei $\mathrm{p} \leq 0,05$ signifikant) // Boxplot of the analytical parameter residual sugar in wine (* boxplots marked with different letters differ significantly with the Tukey-B test at $p \leq 0.05$ ). 


\section{Geruch Allgemein}

Intentität im Geruch

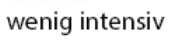

Reintönigkeit im Geruch

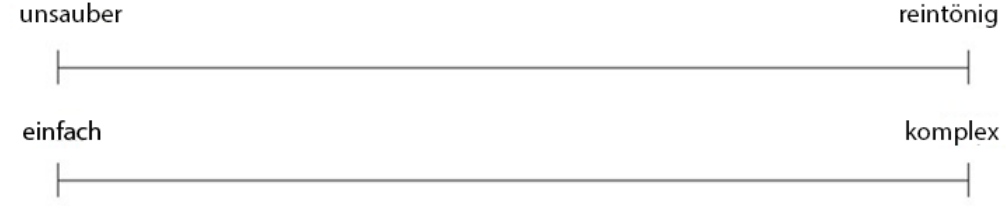

Komplexität

\section{Geruch}

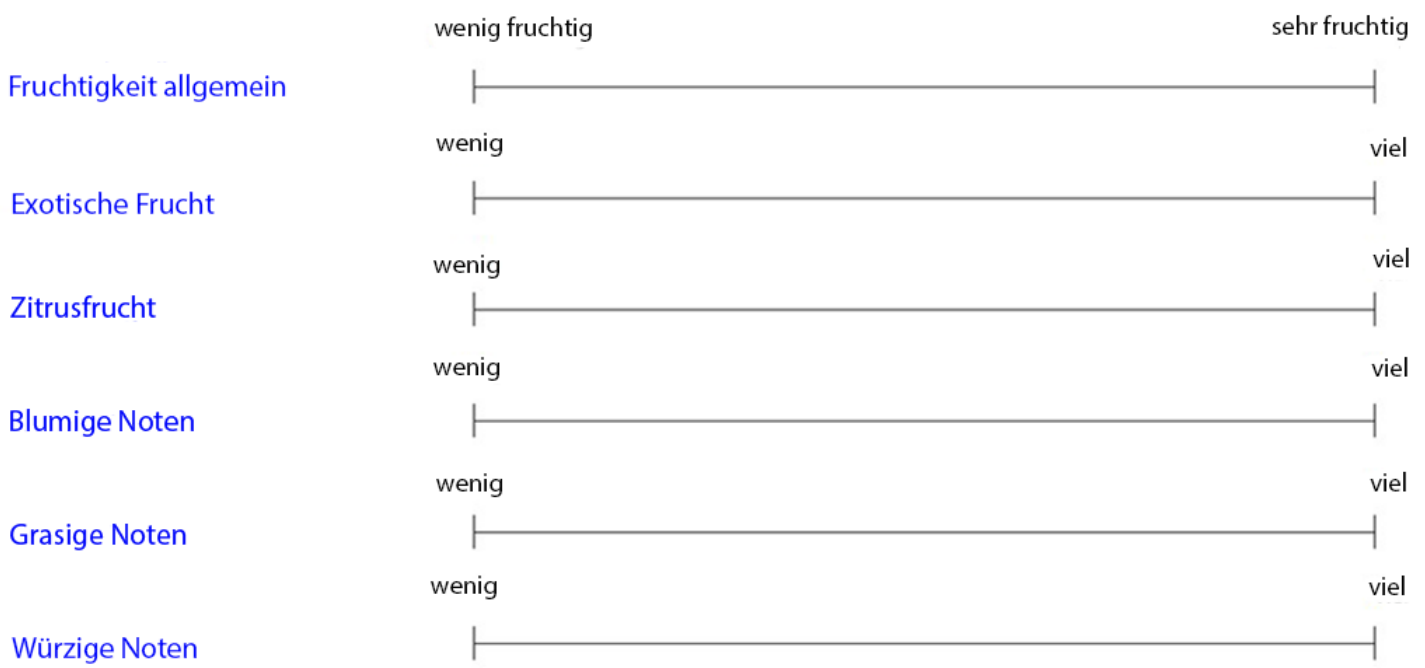

\section{Geruch und Geschmack}

Reintönigkeit im Gaumen

Typizität

Fülle

Harmonie

Gesamteindruck

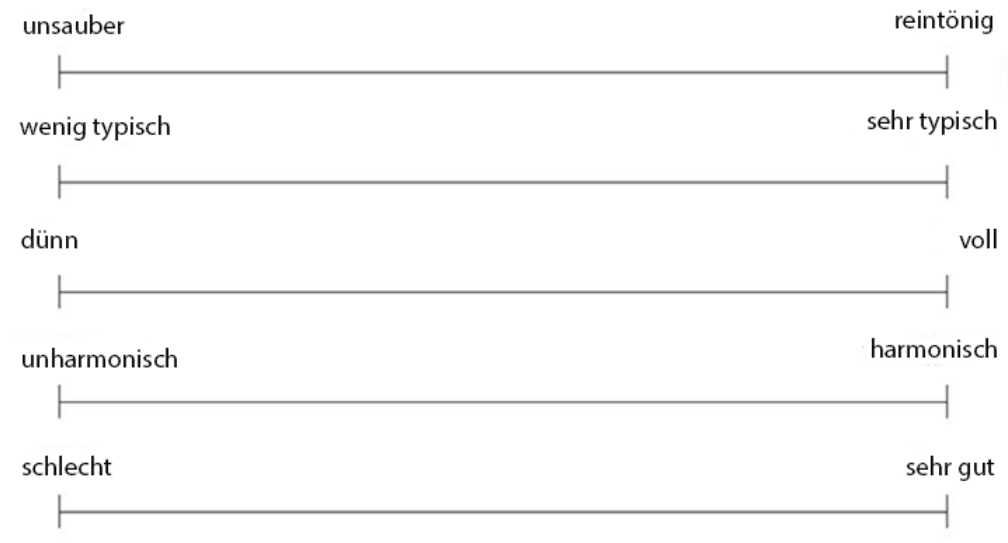

Abb. 2: Verkostungsbogen für sensorische Profilanalyse bei Weissburgunder und Sauvignon Blanc // Tasting sheet for sensory profile analysis on Pinot Blanc and Sauvignon Blanc. 


\section{Sensorial description Riesling vintage 2017}

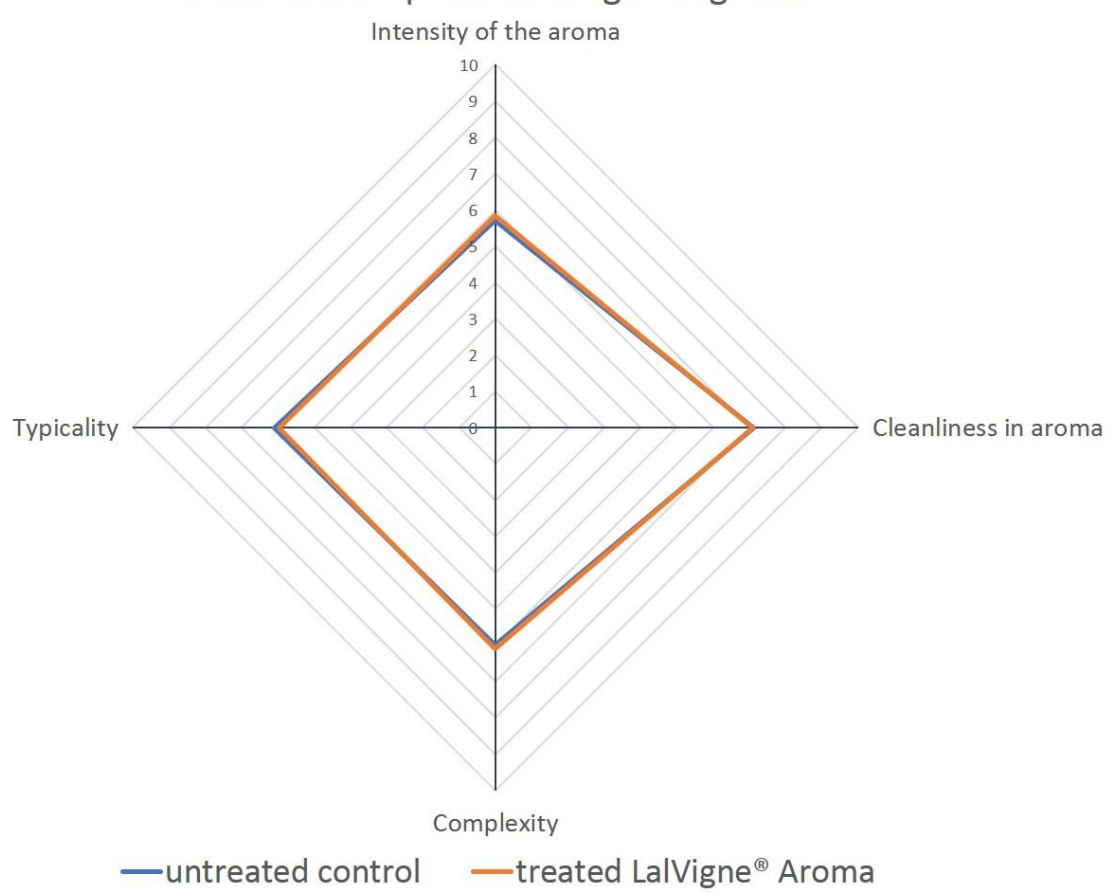

Abb. 3: Spinnennetzdiagramm zur sensorischen Beschreibung Riesling Jahrgang 2017; Vergleich unbehandelte Kontrolle und behandelt LaIVigne ${ }^{\circledR}$ Aroma. ${ }^{*} \mathrm{p} \leq 0.05 ; * * \mathrm{p} \leq 0.01 ; * * * \mathrm{p} \leq 0.001 / /$ Radar chart of the sensorial description Riesling vintage 2017; comparison of untreated control and treated LalVigne ${ }^{\circledast}$ Aroma. ${ }^{*} p \leq 0.05 ;{ }^{* *} p \leq 0.01 ;{ }^{* * *} p \leq 0.001$.

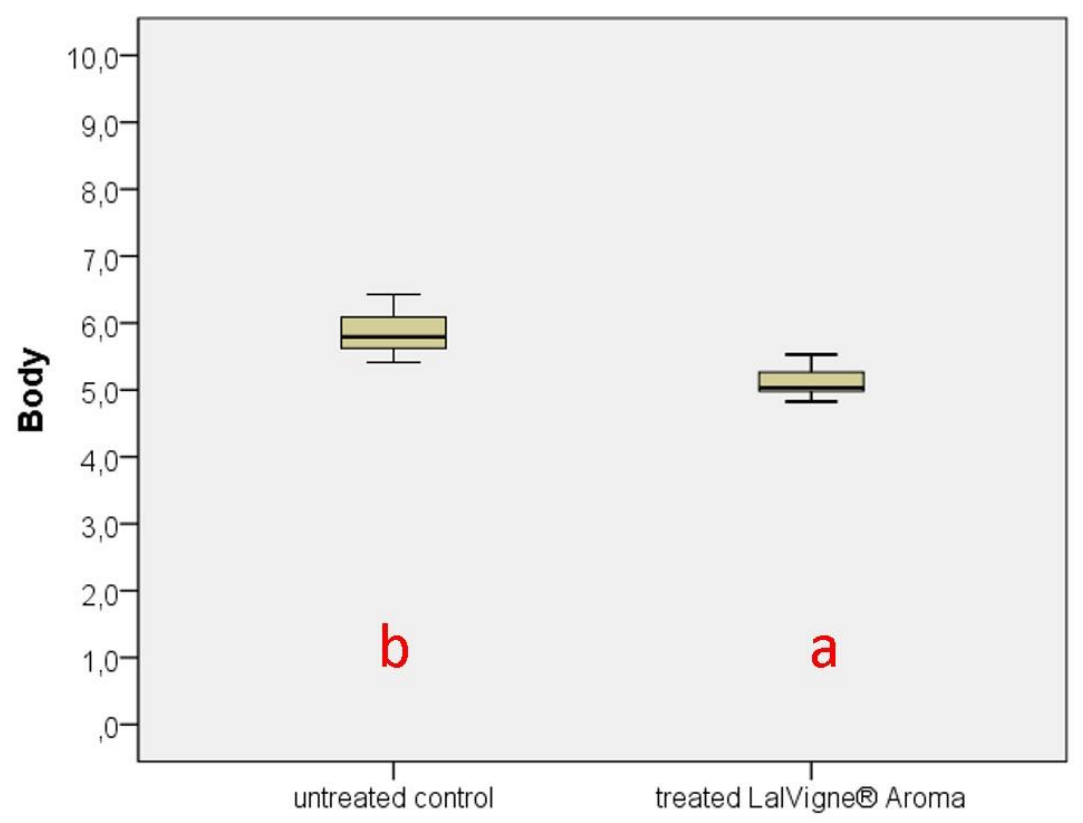

Abb. 4: Boxplot zum sensorischen Parameter Fülle im Wein (*Boxplots welche mit verschiedenen Buchstaben gekennzeichnet sind, unterscheiden sich mit dem Tukey-B Test bei $\mathrm{p} \leq 0,05$ signifikant) // Boxplot of the sensory parameter body in wine (* boxplots marked with different letters differ significantly with the Tukey-B test at $p \leq 0.05)$. 


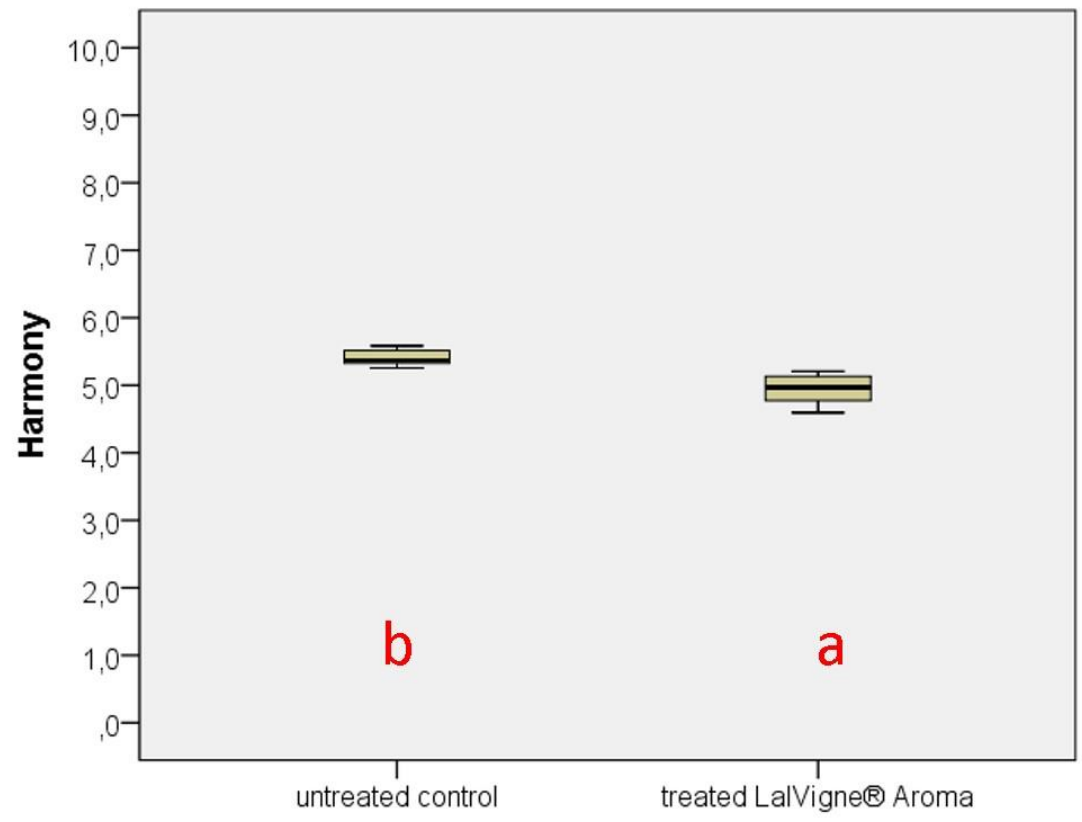

Abb. 5: Boxplot zum sensorischen Parameter Harmonie im Wein (*Boxplots welche mit verschiedenen Buchstaben gekennzeichnet sind, unterscheiden sich mit dem Tukey-B Test bei $\mathrm{p} \leq 0,05$ signifikant) // Boxplot of the sensory parameter harmony in wine (* boxplots marked with different letters differ significantly with the Tukey-B test at $p \leq 0.05)$.

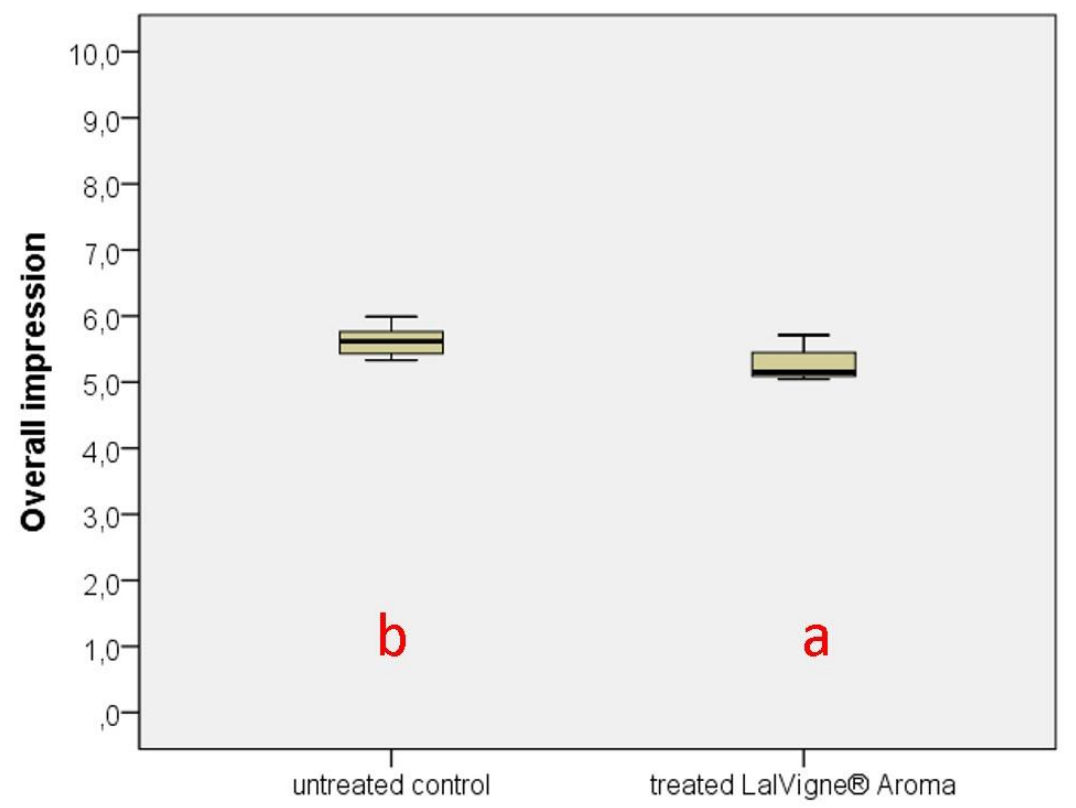

Abb. 6: Boxplot zum sensorischen Parameter Gesamteindruck (*Boxplots welche mit verschiedenen Buchstaben gekennzeichnet sind, unterscheiden sich mit dem Tukey-B Test bei $\mathrm{p} \leq 0,05$ signifikant) // Boxplot of the sensory parameter overall impression (* boxplots marked with different letters differ significantly with the Tukey-B test at $p \leq 0.05$ ). 


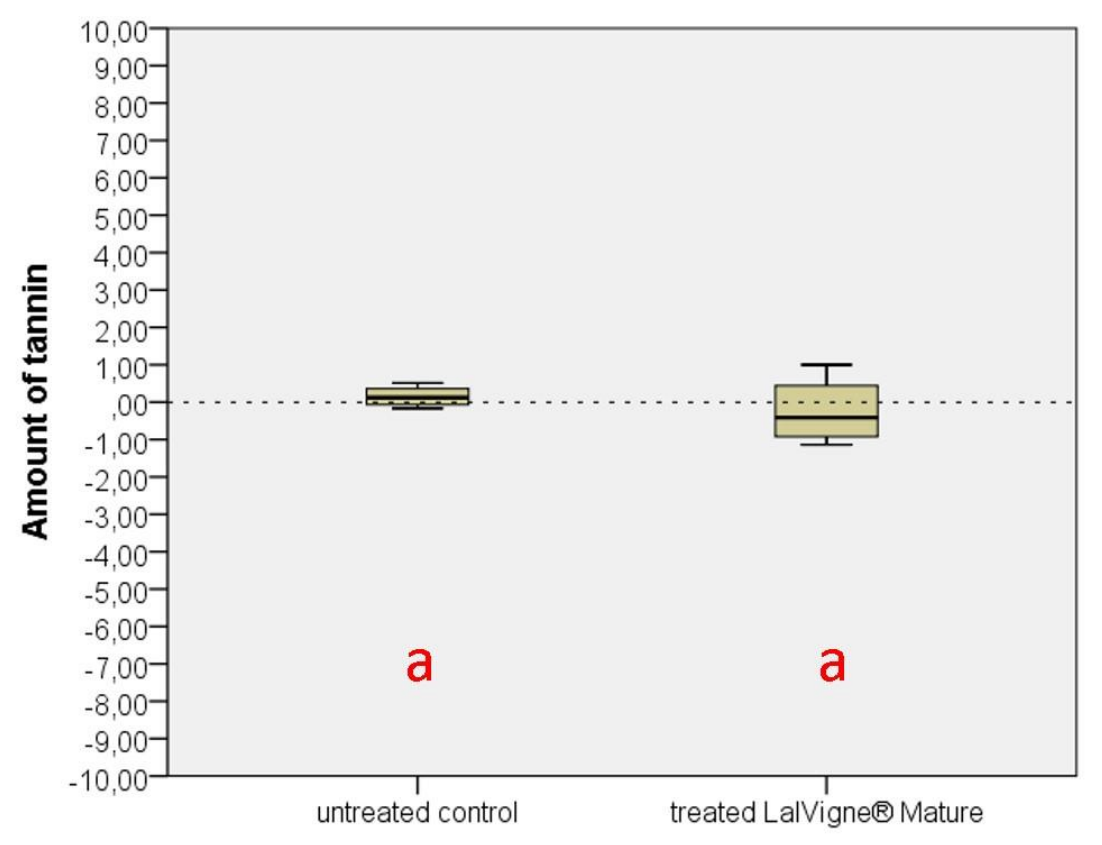

Abb. 7: Boxplot zum sensorischen Parameter Gerbstoffquantität (*Boxplots welche mit verschiedenen Buchstaben gekennzeichnet sind, unterscheiden sich mit dem Tukey-B Test bei $\mathrm{p} \leq 0,05$ signifikant) // Boxplot of the sensory parameter overall impression ( ${ }^{*}$ boxplots marked with different letters differ significantly with the Tukey-B test at $p \leq 0.05$ ).

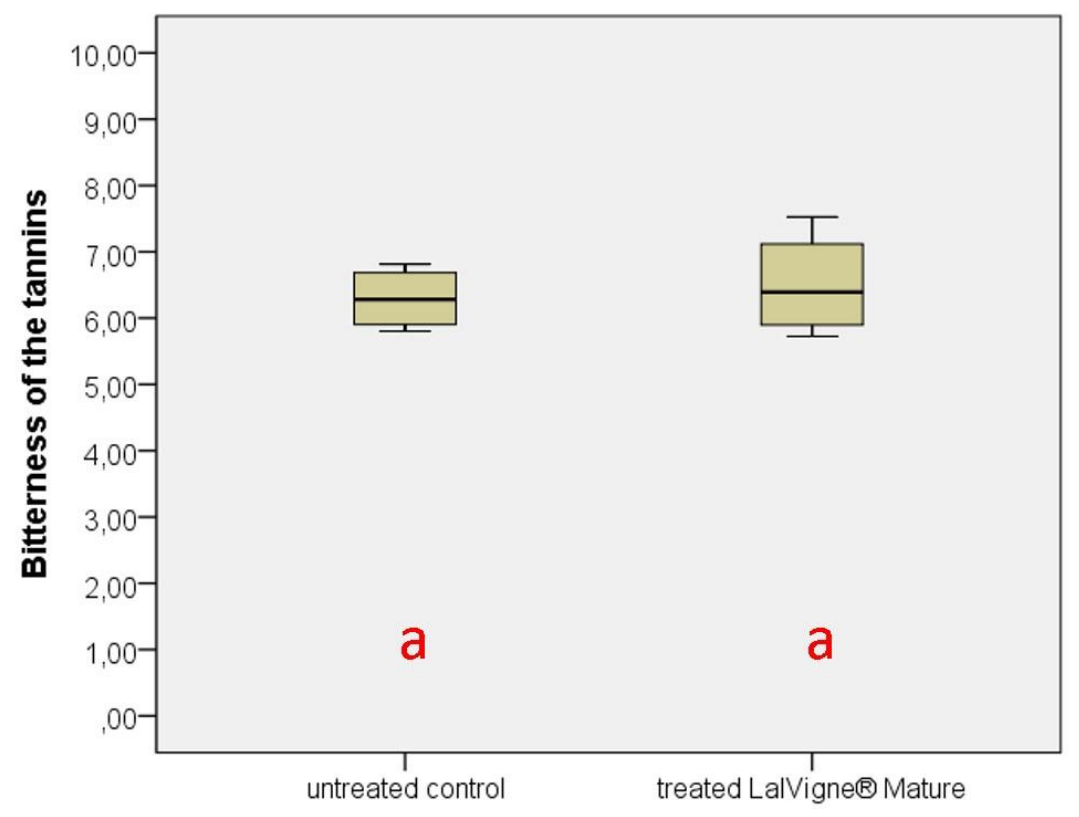

Abb. 8: Boxplot zum sensorischen Parameter Bitterkeit der Gerbstoffe (*Boxplots welche mit verschiedenen Buchstaben gekennzeichnet sind, unterscheiden sich mit dem Tukey-B Test bei $p \leq 0,05$ signifikant) // Boxplot of the sensory parameter Bitterness of the tannins ( ${ }^{*}$ boxplots marked with different letters differ significantly with the Tukey-B test at $p \leq 0.05)$. 


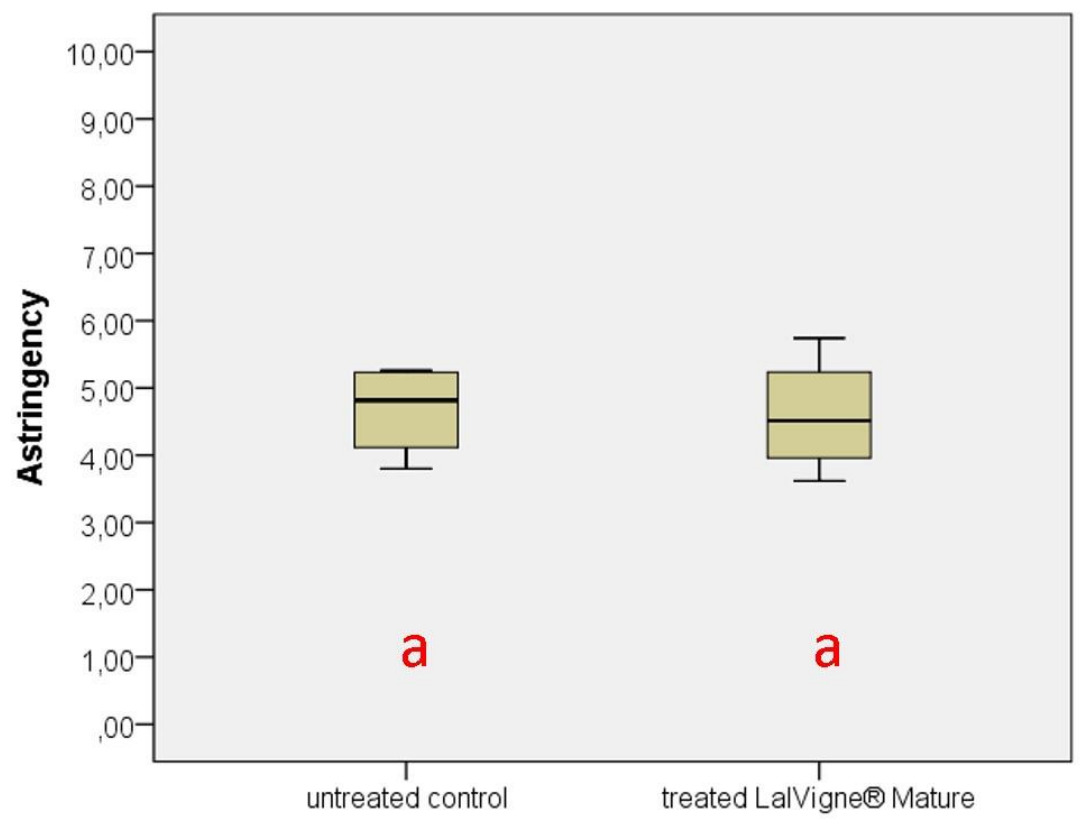

Abb. 9: Boxplot zum sensorischen Parameter Adstringenz (*Boxplots welche mit verschiedenen Buchstaben gekennzeichnet sind, unterscheiden sich mit dem Tukey-B Test bei $\mathrm{p} \leq 0,05$ signifikant) // Boxplot of the sensory parameter Astringency ( ${ }^{*}$ boxplots marked with different letters differ significantly with the Tukey-B test at $p \leq 0.05$ ).

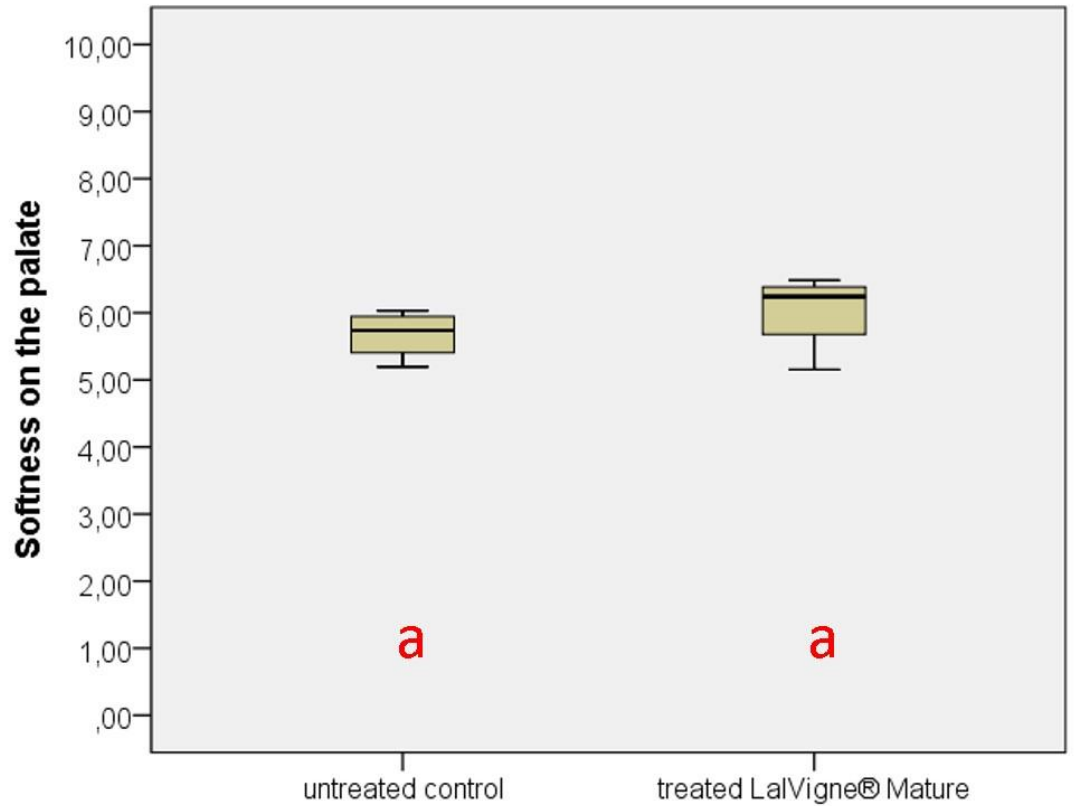

Abb. 10: Boxplot zum sensorischen Parameter Weichheit am Gaumen (*Boxplots welche mit verschiedenen Buchstaben gekennzeichnet sind, unterscheiden sich mit dem Tukey-B Test bei $\mathrm{p} \leq 0,05$ signifikant) // Boxplot of the sensory parameter Softness on the palate (* boxplots marked with different letters differ significantly with the Tukey-B test at $p \leq 0.05)$. 


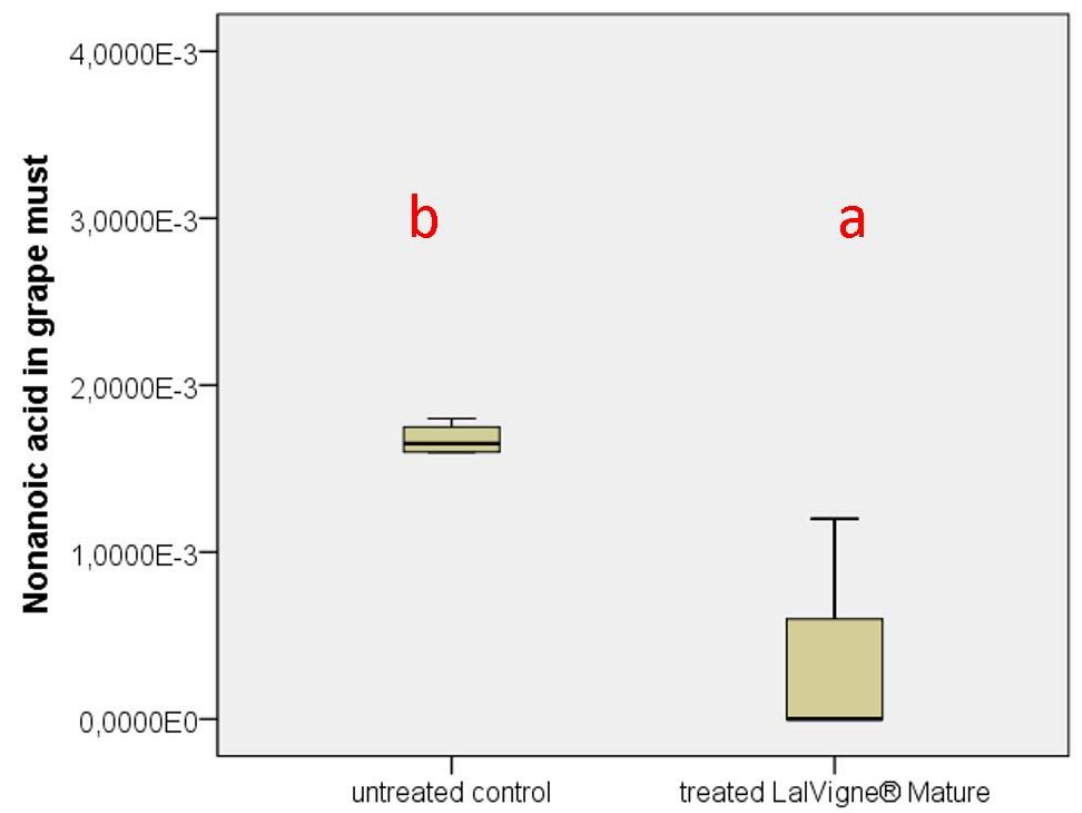

Abb. 11: Boxplot zum analytischen Parameter Nonanoic acid im Traubenmost (*Boxplots welche mit verschiedenen Buchstaben gekennzeichnet sind, unterscheiden sich mit dem Tukey-B Test bei $\mathrm{p} \leq 0,05$ signifikant) // Boxplot of the analytical parameter Nonanoic acid in grape must ( ${ }^{*}$ boxplots marked with different letters differ significantly with the Tukey-B test at $p \leq 0.05$ ).

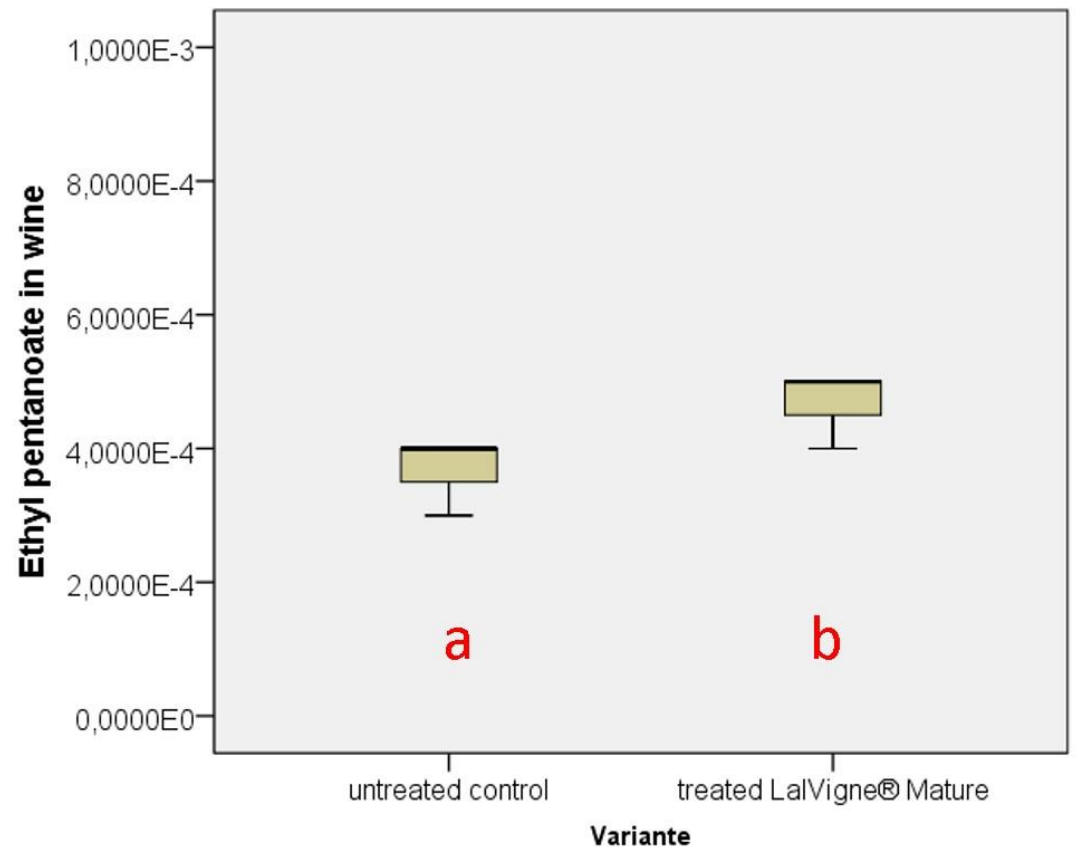

Abb. 12: Boxplot zum analytischen Parameter Ethyl pentanoate im Wein (*Boxplots welche mit verschiedenen Buchstaben gekennzeichnet sind, unterscheiden sich mit dem Tukey-B Test bei $\mathrm{p} \leq 0,05$ signifikant) // Boxplot of the analytical parameter Ethyl pentanoate in wine $\left({ }^{*}\right.$ boxplots marked with different letters differ significantly with the Tukey-B test at $p \leq 0.05$ ). 


\section{(c) (1) (9)}

Dieses Werk ist lizenziert unter einer Creative Commons Namensnennung-Nicht kommerziell 4.0 International Lizenz. Quest'opera è distribuita con Licenza Creative Commons Attribuzione - Non commerciale 4.0 Internazionale.

This work is licensed under a Creative Commons Attribution-NonCommercial 4.0 International License.

Für alle Abbildungen und Tabellen ohne Nennung des Urhebers gilt: @ Versuchszentrum Laimburg. Per tutte le immagini e tabelle senza menzione dell'artefice vale: (c) Centro di Sperimentazione Laimburg. For all figures and tables without mention of the originator applies: (C) Laimburg Research Centre. 